\title{
An alternative pulse classification algorithm based on multiple wavelet analysis
}

\author{
Deniz Ertuncay (D) - Giovanni Costa
}

Received: 1 March 2019 / Accepted: 28 May 2019 / Published online: 26 June 2019

(C) The Author(s) 2019

\begin{abstract}
Near fault ground motions may contain impulse behavior on velocity records. Such signals have a particular indicator which makes it possible to distinguish them from non-impulsive signals. These signals have significant effects on structures; therefore, they have been investigated for more than 20 years. In this study, we used Ricker and Morlet wavelets in order to analyze the wavelet power spectrum of the strong motion signals to investigate the impulsiveness. Both the area around the PGV and the area that exceeds the minimum threshold for the energy function are used in order to determine the position of the pulse. On both of these cases, particular criteria are used in order to characterize the signal. Then, we calculate the pulse period of the pulse region. Ricker and Morlet wavelets are also used to mimic the pulse signal. This method provides advanced information about the position of the maximum energy of the pulse part of the signal. We found that the impulsive part of the signal is frequently at the position where PGV occurs and the Ricker wavelet is better than the Morlet wavelet on mimicking the pulse part of the waveform. Spectral responses of
\end{abstract}

D. Ertuncay $(\bowtie) \cdot$ G. Costa

Department of Mathematics and Geosciences,

University of Trieste, SeisRaM group, Trieste, Italy

e-mail: deniz.ertuncay@phd.units.it

G. Costa

e-mail: costa@units.it strong motion waveform and the wavelets have strong correlation at around pulse period. Results show consistency with previous studies; hence, it can be used as a solid alternative on pulse shape signal investigations.

Keywords Near-fault ground motion · Pulse-like ground motion - Velocity pulse - Wavelet analysis . Pulse shaped signal extraction

\section{Introduction}

The increasing number of seismic Stations has allowed the study of near-fault earthquake ground motions in seismology. Occasionally, Stations recorded earthquake signals with unexpected patterns. These ground motions, called henceforward pulseshape signals, can be seen at the beginning of the earthquake signal in velocity records.

Pulse-shape signals are researched in both classical and engineering seismology. In engineering seismology, it is vital to identify pulse-shape signals, since they can create high demands on structures around the period of the pulse signal (Bertero et al. 1978; Anderson and Bertero 1987; Hall et al. 1995; Iwan 1997; Alavi and Krawinkler 2001; Menun and Fu 2002; Makris and Black 2004; Mavroeidis et al. 2004; Akkar et al. 2005; Luco and Cornell 2007; Kalkan and Kunnath 2006). However, some of the probabilistic seismic hazard analysis (PHSA) models (Lanzano et al. 2016) and building design codes do not consider their 
effects on PHSA models. Although it is a rare phenomenon, it is vital to investigate pulse-shape signals due to their hazardous effects.

Pulse-shape signals can appear in some earthquake scenarios, like those considering forward directivity, which occurs when receivers are located in the forward direction of the fault rupture (Somerville et al. 1997; Somerville 2003, 2005; Spudich and Chiou 2008), the fling step effect, which is a permanent displacement of the ground resulting from fault rupture (Mavroeidis and Papageorgiou 2002) and when rupture velocity and shear-wave velocity of the bedrock of the site of interest are similar.

The seldom occurrence of pulse-shape signals depends mostly on the above conditions. However, because of their scarcity, velocity pulses are not taken into account in most of the ground motion prediction equations (GMPE) (Abrahamson et al. 2016; Boore et al. 2014). Yet, standard and median deviations of GMPE can be calibrated with certain factors to involve the effect of a pulse-shape signal (Akkar and Cheng 2016).

Indicators of pulse-shape signals in the waveforms are:

1. Signals with long and large amplitudes (Somerville et al. 1997),

2. A high PGV/PGA ratio (Bray and RodriguezMarek 2004),

3. Earthquake energy concentrated on one (or a few) pulse(s) (Somerville et al. 1997),

4. Unexpectedly high response values at the pulse period on response spectra (Yang and Wang 2012).

Various methods have been created for identifying the pulse-shape signals. Mavroeidis and Papageorgiou (2003) proposed a wavelet analysis to construct a mathematical representation of the pulse, which depends on amplitude, period, duration, and phase shift. Shahi and Baker (2014) used a 4th-order Daubechies wavelet to determine pulse-shape signals. The method has some constraints such as a minimum PGV amplitude, a pulse arrival located at the beginning of the signal and arbitrary thresholds for the energy function. Mena and Mai (2011) used windowed Fourier transform analysis for the pulse shape signal and its position with certain energy thresholds. Chang et al. (2016) used the energy function with certain thresholds to determine the pulse-shape signal position and period. Ghaffarzadeh (2016) used the S-transform to identify the pulses. Kardoutsou et al. (2017) used a cross-correlation between the potential pulse-shape signal and the wavelet functions to determine the pulse shape. Methods of Shahi and Baker (2014) and Chang, Chang et al. (2016) are explained in detail in Section 3.

The goal of this study is to create an alternative pulse identification algorithm. Main considerations are determining the time location of the pulse and mimicking impulsive part of the signal with known wavelets. Ricker and Morlet wavelets are used both for spectrum analysis to determine the pulse period and the region with maximum energy and mimicking impulsive part of the signal. Waveform that are identified as pulse shaped are compared with the wavelets which are created with calculated pulse period by checking spectral responses. If the wavelets correspond to the features of the long period part of the earthquake signal, the algorithm is considered as successful.

\section{Data}

The analyzed ground motions are selected from NGAWest2 (Ancheta et al. 2012), GeoNet, Itaca (Pacor et al. 2011; Luzi et al. 2016), and K-Net databases, which contain data from crustal earthquakes. Earthquake signals that are recorded due to $M w \geq$ 5.5 earthquakes with a maximum distance range of $150 \mathrm{~km}$ from the epicenter are selected. In order to study pulse-shape signals, East and North components are rotated to radial and transverse components. Acceleration waveform has been bandpass filtered between 0.05 and $10 \mathrm{~Hz}$ and integrated to obtain velocity waveform. In total, our database contains 2785 waveform.

\section{Previous methods}

Two aforementioned algorithms, Shahi and Baker (2014) and Chang et al. (2016), are used for comparison with our new method. These algorithms are chosen since both are well known and widely used when dealing with this topic. 


\subsection{Shahi and Baker (2014)}

Shahi and Baker (2014) classification algorithm uses wavelet-based signal processing to detect pulse-shape signals in the area with the largest value of velocity (PGV). The algorithm can differentiate early and late arrival pulses by analyzing the arrival of PGV. Early arrivals of PGV generally indicate directivity effects.

Classification algorithm uses two criteria to determine whether the signal has impulsive or nonimpulsive behavior. First criterion is the hazardousness of the signal. If PGV is less than $30 \mathrm{~cm} / \mathrm{s}$, it is considered as non-hazardous signal. Second criterion is that the pulse indicator (PI) values should be bigger than 0. Calculation of PI has two stages. In the first stage, principal component (PC) is found as explained in Eq. 1:

$$
P C=0.63 \times(P G \text { Vratio })+0.777 \times(\text { energyratio })
$$

PGVratio indicates the ratio of the PGV value of the residual signal and the PGV value of the original signal, which is calculated by subtracting the original signal from that produced by the 4th-order Daubechies wavelet signal. energyratio is the ratio calculated by dividing the power of the residual signal by the power of the original signal. In the second stage, PI is calculated as Eq. 2:

$$
\begin{aligned}
P I= & 9.384(0.76-P C-0.00616 P G V) \\
& \times\left(P C+6.914 \times 10^{-4} P G V-1.072\right)-6.179
\end{aligned}
$$

If the signal is considered as pulse shaped signal, then $4^{\text {th }}$ order Daubechies wavelet is fitted to entire waveform. It is hard to determine where the impulsive part of the signal starts and ends by using Shahi and Baker (2014) classification algorithm.

\subsection{Chang et al. (2016)}

Chang et al. (2016) use an energy-based classification algorithm. The algorithm determines a region around the PGV and determines the energy ratio between the pulse region and the total energy of the signal by taking the squared values on both signals. The region around PGV is calculated by using a least-square fitting for various pulse periods; then, the one with the smallest residual is used for the pulse region. The energy ratio is then calculated as Eq. 3:

$E(t)=\frac{\int_{t_{\mathrm{s}}}^{t_{\mathrm{e}}} v^{2}(\tau) d \tau}{\int_{0}^{\infty} v^{2}(\tau) d \tau}$

$t_{\mathrm{S}}$ and $t_{\mathrm{e}}$ represent the starting and ending point of the impulse part in time axis and $v^{2}$ represents the velocity time history of the signal. If the ratio between the pulse region energy, the numerator part of Eq. 3, and the total energy, the denominator of Eq. 3, exceeds 0.34 , the signal is considered as a pulse-shaped signal.

If the signal is considered as pulse shaped signal, then a waveform is fitted to impulsive part of signal. Contrary to Shahi and Baker (2014), one can identify the impulsive part of the seismic signal by using the algorithm of Chang et al. (2016).

\section{The new method}

Previous attempts to determine pulse shape signals were concentrated on determining if the signal has an impulsive or non-impulse behavior. Another common goal is to determine the period of the pulse, since it can have significant effects on structures. Therefore, the pulse period has been focused on when mimicking the pulse signal. One of the main assumptions on pulse shape signals is that the impulsive part is where PGV is located.

In this method, we took the minimum threshold of PGV as $30 \mathrm{~cm} / \mathrm{s}$, if the pulse occurs where PGV has occurred. As proposed by Shahi and Baker (2014), we used wavelet analysis to determine pulse shape signals. Unlike previous studies, apart from the PGV time interval, we also considered the possibility that the pulse occurs at other time intervals of the earthquake signal.

Mavroeidis and Papageorgiou (2003) and Chang et al. (2016) focused on the impulsive part of the signal for analysis whereas Shahi and Baker (2014) fitted almost all of the signal. We focused only on the impulsive part of signal, similar to Mavroeidis and Papageorgiou (2003) and Chang et al. (2016). Main goal of this study is to create a robust alternative to 
identify the pulse shaped signals. We implemented threshold for PGV and wavelet analysis for signal process to have similarities with previous studies while adding new features that these studies did not consider.

\subsection{Wavelet analysis}

Wavelet analysis package of Torrence and Compo (1998) is used for signal analysis. Two different wavelet types, which are Ricker (Appendix 1) and Morlet wavelet (Appendix 2), are implemented to the wavelet analysis process. Wavelet power spectrums of the signals are calculated by using these wavelets. Morlet wavelet is complex, while Ricker is real-valued. The complex wavelet function returns both amplitude and phase information, whereas real wavelet function returns only real components. This allows to isolate discontinuities. Since both of the wavelets are giving the same qualitative results on power spectra, both of them can be treated as equal. However, Ricker can distinguish the discontinuities, since it is a real-valued function, whereas Morlet can give more smooth results, which is important when there is a high-frequency content in the pulse region (Fig. 1).

The resolution of the wavelet function depends on the width of real space and width in Fourier space. A broad function will give a poor time resolution but a good frequency resolution, and vice versa. The width of the wavelet is proportional to the sampling rate of the signal.

As a result of the analysis, one can determine the power spectrum values over time. The maximum power spectrum values at PGV and the biggest power spectrum value of the signal (if it does not occur at PGV) are used in the pulse identification.

\subsection{Pulse identification}

Unlike previous studies, our method can identify velocity pulses that occur away from the time interval where the PGV is located. Several decision mechanisms are used to identify pulse shape signals. The criteria for pulse shape signals differ with respect to the position of the pulse, as explained in Section 4.2.1 and in Section 4.2.2.

\subsubsection{Velocity pulse at $P G V$}

Most of the seismic energy is assumed to be concentrated at the position where PGV occurs. One of the logical ways to analyze the signal is to focus around the region of PGV. Our method is similar to Chang et al. (2016) since our method is also looking for the energy ratio of the waveform. Furthermore, our method is also looking for the spectral energy, which is similar to the method of Mena and Mai (2011). The threshold of our method occurs when the average of these two parameters around the PGV are equal or bigger than $30 \%$ of the whole waveform. The criteria are reported below:

1. $\mathrm{PGV} \geq 30 \mathrm{~cm} / \mathrm{s}$.

2.

$$
\frac{\left(\frac{\int_{t_{\mathrm{s}}}^{t_{\mathrm{e}}} v^{2}(\tau) d \tau}{\int_{0}^{\infty} v^{2}(\tau) d \tau}+\frac{\int_{t_{\mathrm{s}}}^{t_{\mathrm{e}}} W P S(\tau) d \tau}{\int_{0}^{\infty} W P S(\tau) d \tau}\right)}{2} \geq 0.30
$$

In Eq. $4, t_{\mathrm{s}}$ and $t_{\mathrm{e}}$ represent the starting and ending points of the pulse, respectively. These points are found by identifying the period $\left(T_{\mathrm{p}}\right)$ where the maximum wavelet power spectrum occurs at PGV. The pulse area is then identified as $t_{P G V} \mp T_{p} / 2$ where $t_{P G V}$ represents the time of the PGV. WPS indicates the wavelet power spectrum. The parameters can be seen in Fig. 2.

The left side of the numerator in Eq. 4 indicates the energy ratio between the impulsive part (velocity time history between $t_{\mathrm{s}}$ and $t_{\mathrm{e}}$ in time axis). The right side of the numerator is the ratio between wavelet spectrum energy of the waveform and impulsive part between the aforementioned area of the signal. Integrals are for summation process and infinity signs indicate the whole waveform.

\subsubsection{Velocity pulse outside the PGV region}

Unlike previous studies, we also checked for the biggest energy arrival rather than the position of PGV. The logic behind the energy calculation is the same as in the Section 4.2.1. The minimum amplitude is fixed at $25 \mathrm{~cm} / \mathrm{s}$. However, this amplitude is not the amplitude of PGV, but the biggest amplitude of the region 
Fig. 1 Velocity waveform (upper), Ricker wavelet power spectrum (center) and Morlet wavelet spectrum (lower) of 1992 Landers earthquake $\left(M_{\mathrm{w}}=7.3\right)$, Yermo Fire Station (Epicentral distance $\left.\left(r_{\mathrm{ep}}\right)=85.99 \mathrm{~km}\right)$. Red and blue colors represent high and low concentration of power, respectively
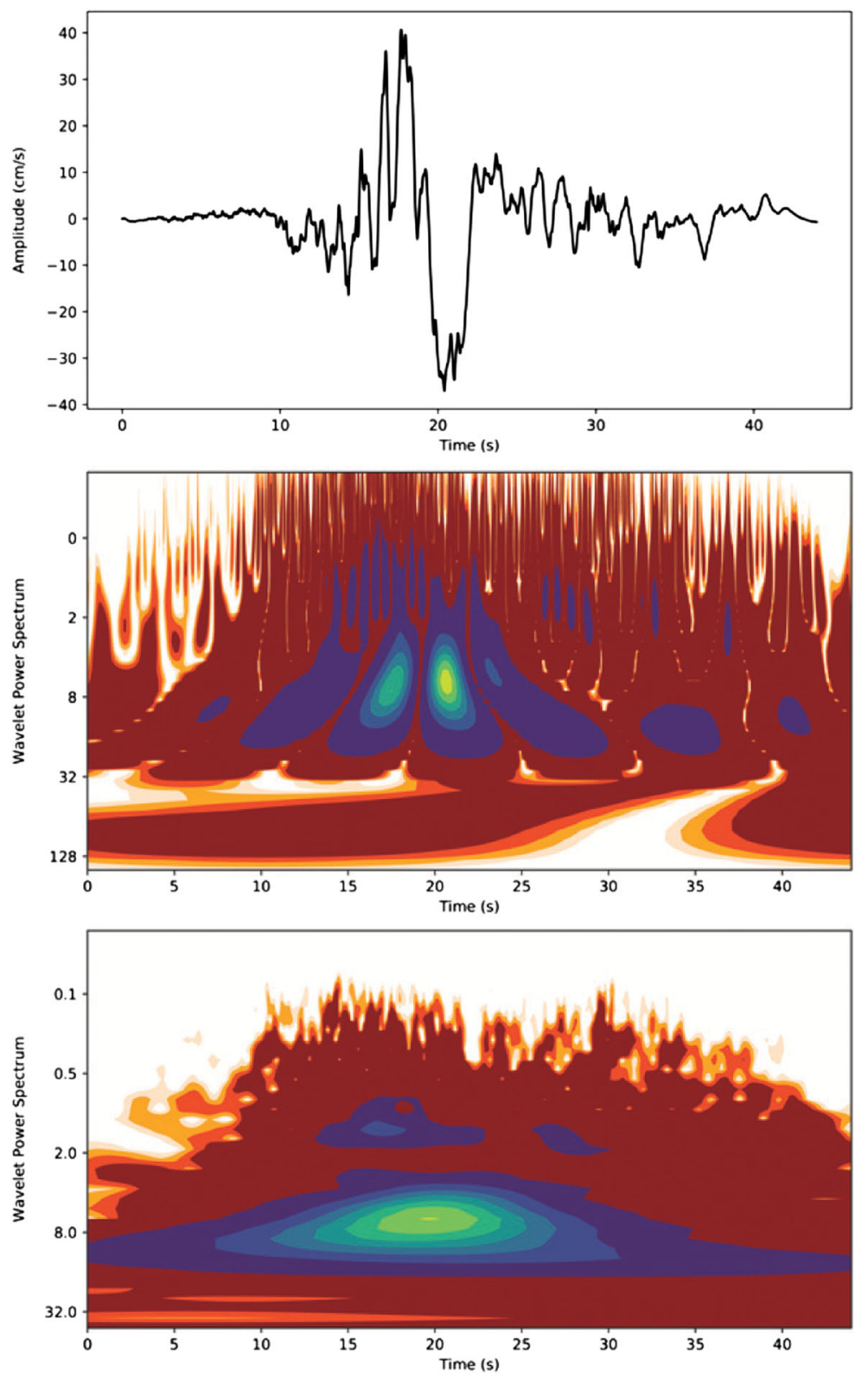

where the maximum energy is concentrated. Furthermore, the maximum energy of the region should be equal or bigger than $10 \%$ of the energy of the PGV region. The average of the energy of the waveform and wavelet power spectrum of this region should exceed $30 \%$ of the total energy of the signal. The criteria are reported below:

1. The biggest amplitude, in absolute sense, in the area where the maximum power spectrum value occurred, should be equal or bigger than $25 \mathrm{~cm} / \mathrm{s}$.
2. Difference between PGV and the time where the maximum power spectrum in time axis should be larger than $T_{p} / 4$.

3.

$$
\frac{\int_{t_{\mathrm{semax}}}^{t_{\mathrm{eemax}}} v^{2}(\tau) d \tau}{\int_{t_{\mathrm{s}}}^{t_{\mathrm{e}}} v^{2}(\tau) d \tau} \geq 1.1
$$

4.

$$
\frac{\int_{t_{\mathrm{semax}}}^{t_{\mathrm{eemax}}} W P S(\tau) d \tau}{\int_{t_{\mathrm{s}}}^{t_{\mathrm{e}}} W P S(\tau) d \tau} \geq 1.1
$$




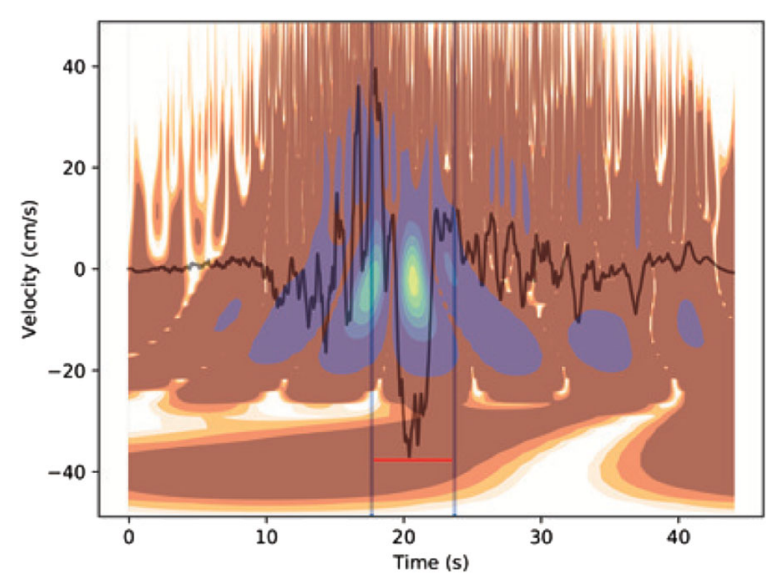

Fig. 21992 Landers earthquake, Yermo Fire Station velocity waveform. Red line and blue lines represent width $\left(T_{\mathrm{p}}\right)$ and borders $\left(t_{\mathrm{s}}\right.$ and $\left.t_{\mathrm{e}}\right)$ of the pulse region, respectively. Background image is Ricker wavelet power spectrum of the signal with the same color content of Fig. 1

5.

$$
\frac{\left(\frac{\int_{t_{\mathrm{semax}}}^{t_{\mathrm{eemax}}} v^{2}(\tau) d \tau}{\int_{0}^{\infty} v^{2}(\tau) d \tau}+\frac{\int_{t_{\mathrm{semax}}}^{t_{\mathrm{eemax}}} W P S(\tau) d \tau}{\int_{0}^{\infty} W P S(\tau) d \tau}\right)}{2} \geq 0.30
$$

In Eqs. 5 and 6, $t_{\text {eemax }}$ and $t_{\text {semax }}$ represent the starting and ending points of the pulse in the maximum energy area in time axis. These points are found by identifying, in the area where the maximum power spectrum values are located, the maximum pulse period $\left(T_{\mathrm{p}, \text { emax }}\right)$ of the signal. The pulse area is then identified as $t_{\text {eemax }} \mp T_{\mathrm{p} \text {,emax }}$ where $t_{\mathrm{eemax}}$ represents the time of the biggest value in the $T_{\mathrm{p} \text {,emax }}$ region. The parameters can be seen in Fig. 3 .

Equations 5 and 6 describe the threshold for the energy ratios between the area around the PGV and the area around the maximum energy, if exists, for waveform and wavelet power spectrum, respectively. Other parameters have the same meanings that are explained in Section 4.2.1.

Energy ratio between PGV region and maximum energy region is determined by trial and error method. Time gap of $T_{\mathrm{p}}$ between PGV region and maximum energy region is implemented since Ricker wavelet power spectrum can identify discontinuities and that may cause erroneous interpretation of a single pulse into two or more separate pulses depending on the period. Amplitude threshold of the maximum power

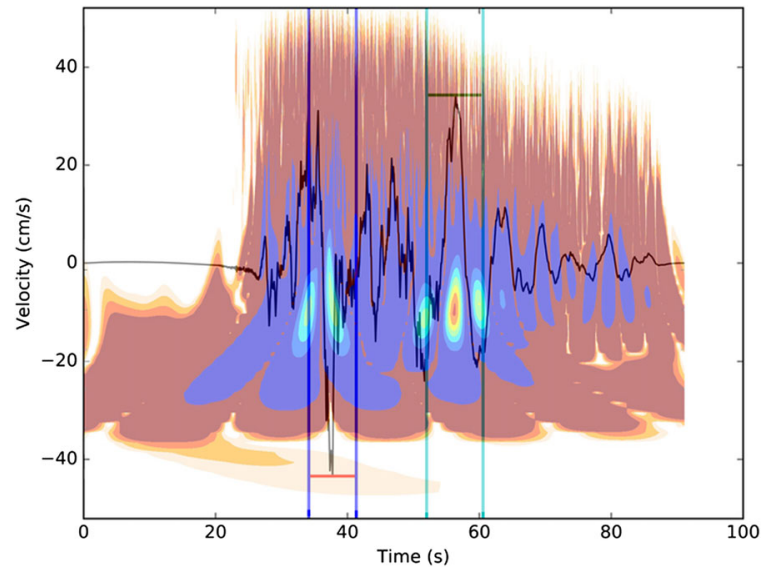

Fig. 31999 Chi-Chi Taiwan Earthquake $\left(M_{\mathrm{w}}=7.6\right)$, TCU051 Station $\left(r_{\mathrm{ep}}=38.53 \mathrm{~km}\right)$ velocity waveform. Red line and blue lines represent the width $\left(T_{\mathrm{p}}\right)$ and borders $\left(t_{\mathrm{s}}\right.$ and $\left.t_{\mathrm{e}}\right)$ of the pulse region around $\mathrm{PGV}$, respectively. The green line and cyan lines represent the width $\left(T_{\mathrm{p}, \mathrm{emax}}\right)$ and borders $\left(t_{\mathrm{eemax}}\right.$ and $\left.t_{\mathrm{semax}}\right)$ of the area where the maximum energy is concentrated, respectively. Background image is Ricker wavelet power spectrum of the signal with the same color content of Fig. 1

spectrum region is selected by considering the same idea behind the $30 \mathrm{~cm} / \mathrm{s}$ of PGV, which is the possibility of creating damages on structures.

Both Ricker and Morlet wavelets are fitted to the pulse region when the algorithm detects a pulse shape signal.

\section{Results}

There are four main features of the pulse-shape signals as explained in Section 1. In this study, we mostly focused on the position, amplitude, and period of the pulse. One way to determine the validity of the method is to compare spectral response of the original and created signals. An unusual spectral response graph is also an indicator of the pulse shape signal. The wavelet signal is expected to imitate the behavior around the pulse period. In order to do that, we visually compared spectral responses of the original strong motion data and the wavelet that is expected to mimic the pulse. Some of the spectral response graphs can be seen in Fig. 4.

We also created a method to check the phase of the impulsive part of the velocity waveform. Impulsive 

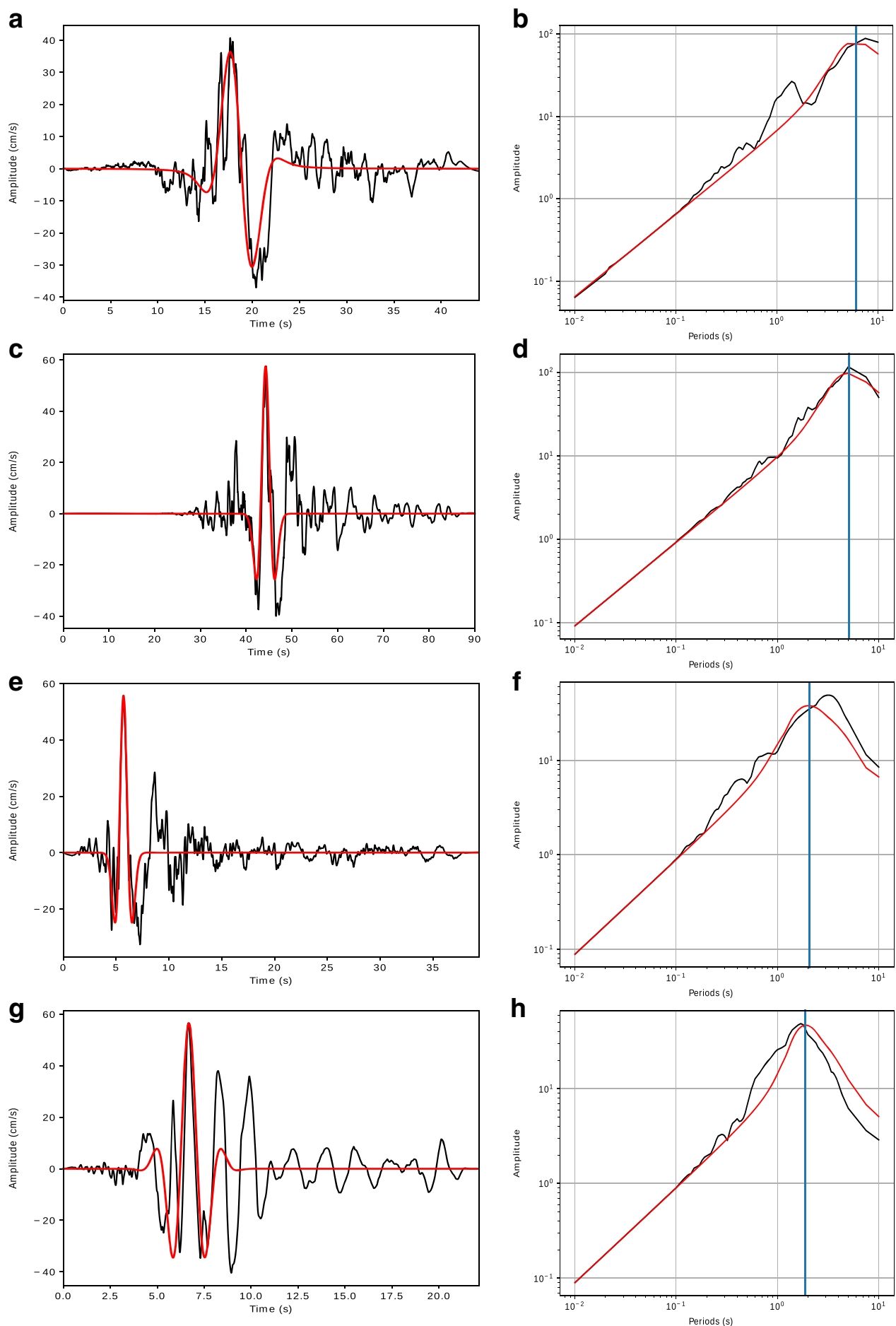

Fig. 4 Velocity waveform and fitted wavelets (left column) and pseudo spectral velocity graphs (right column) of 1992 Landers Earthquake, Yermo Fire Station signal, and obtained Ricker wavelet signal (a, b); 1999 Chi-Chi Taiwan Earthquake, TCU039 Station signal; and obtained Ricker wavelet signal $(\mathbf{c}, \mathbf{d}), 1980$ Irpinia Earthquake $\left(M_{\mathrm{w}}=6.9\right)$, STN Station $\left(r_{\mathrm{ep}}=\right.$
$30.35 \mathrm{~km}$ ) signal and obtained Ricker wavelet signal (e,f) and 1994 Northridge Earthquake $\left(M_{\mathrm{w}}=6.7\right)$, SCE Station $\left(r_{\mathrm{ep}}=\right.$ $24.97 \mathrm{~km}$ ) signal and obtained 3rd-order Morlet wavelet signal $(\mathbf{g}, \mathbf{h})$. In all figures, the blue line represents the period of the pulse. Red and black colors indicate the velocity waveform and fitted wavelet signal, respectively 
signals that can be identified with Ricker wavelet is analyzed, since Ricker wavelet dominated the representation of the impulsive part of the signals. Ricker wavelet can be fitted to the original waveform to visualize the impulsive part more easily, however it is not providing further information about the impulsive part. Method for phase determination is explained in Appendix 3.

Two hundred twenty-nine waveforms out of 2738 waveforms have been identified as pulse-shape signals. Shahi and Baker (2014) and Chang et al. (2016) identified 225 and 229 waveform as pulse-shape signals, respectively. One hundred seventy-eight of the signals are identified as pulse-shape signals by three of these studies, whereas 196 signals are identified as pulse-shape signals by both Shahi and Baker (2014)
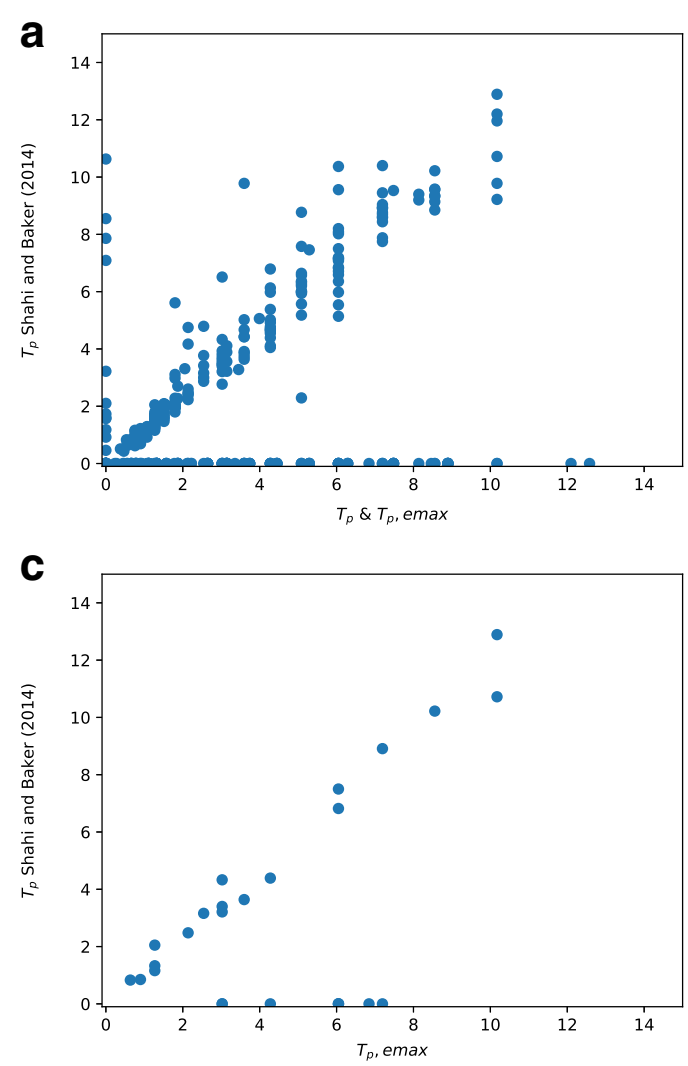

Fig. 5 Pulse periods determined by Shahi and Baker (2014) and this study (a), pulse periods determined by Chang et al. (2016) and this study (b), pulse periods of the signals in which impulsive signals are outside of the PGV region determined by Shahi and Baker (2014) and this study (c), pulse periods of the signals in which impulsive signals are outside of the $\mathrm{PGV}$ region and this study (Fig. 5) and 198 signals are identified as pulse-shape signals by Chang et al. (2016) and this study. Twenty-six of the pulses are located outside the region of PGV. We also checked where the impulse part occurred in the signal and which wavelet better explains the pulse region. Two hundred twenty-six of the pulse-shaped signals are mimicked better by using a Ricker wavelet, whereas only 3 of them are mimicked better by using a 3rd-order Morlet wavelet. A 4th-order Morlet wavelet is not suitable to mimic any of the pulse-shape signals.

\subsection{Comparison with previous studies}

Pulses that are also occurred outside of the PGV region are partially also detected by Shahi and Baker
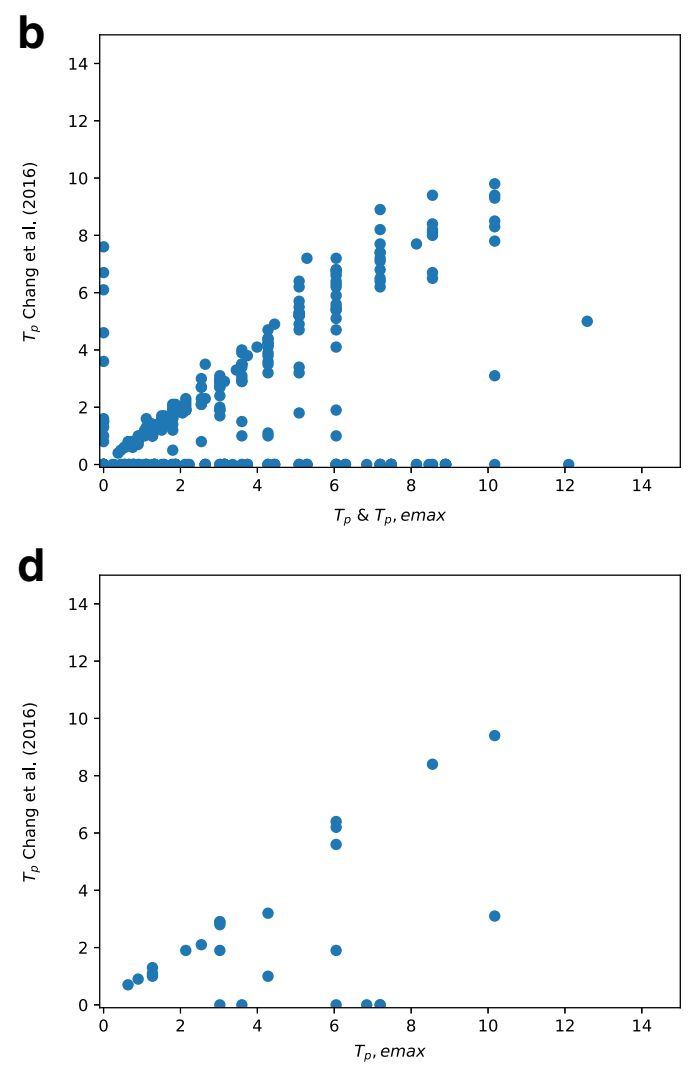

determined by Chang et al. (2016) and this study (d). In panels $\mathbf{a}$ and $\mathbf{b}$, both periods of velocity pulses at PGV $\left(T_{\mathrm{p}}\right)$ and periods of velocity pulses at other places $\left(T_{\mathrm{p}, \mathrm{emax}}\right)$ are plotted. In panels $\mathbf{c}$ and $\mathbf{d}$, only the periods of velocity pulses at other places $\left(T_{\mathrm{p}, \mathrm{emax}}\right)$ are plotted 

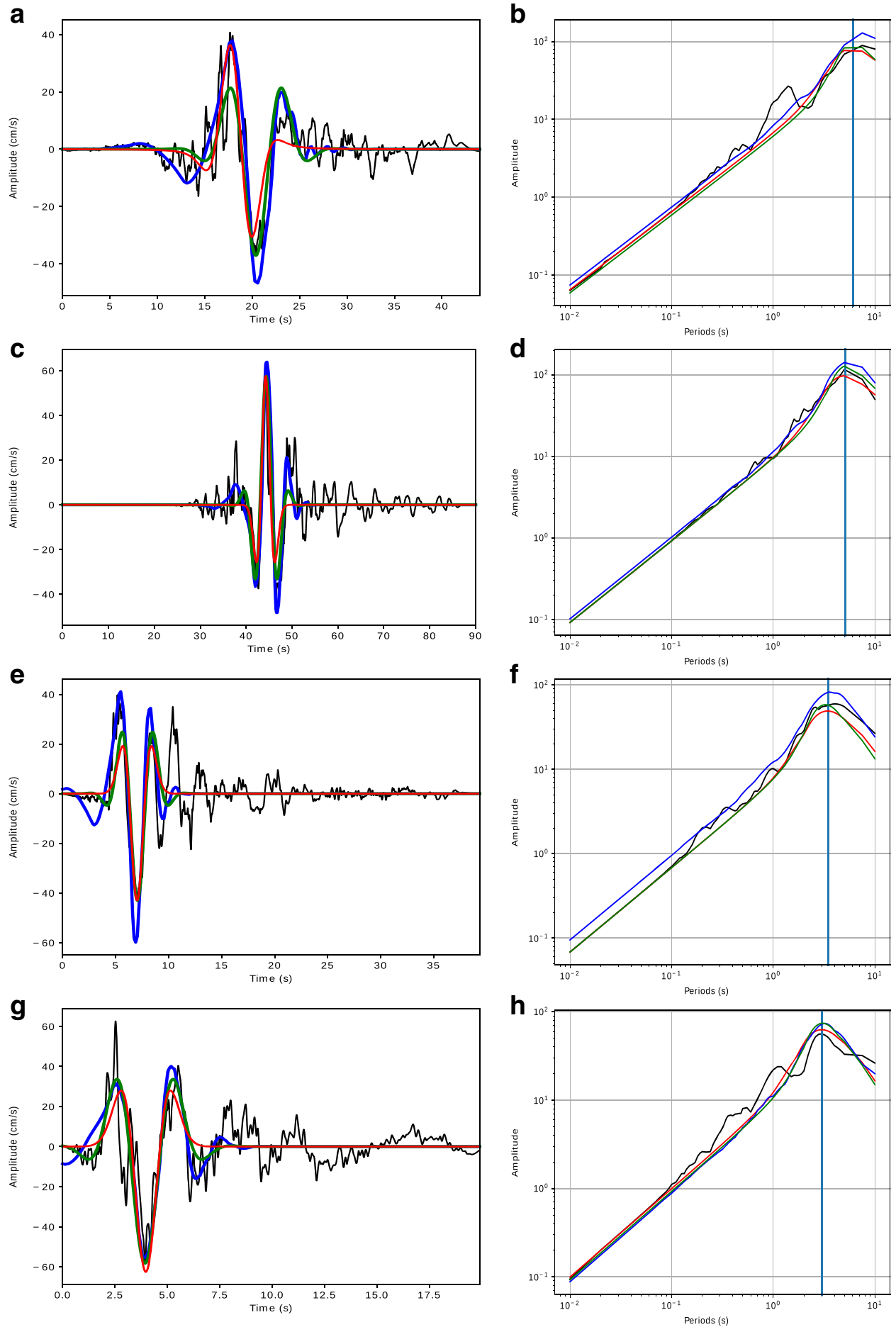

Fig. 6 Velocity waveform and fitted wavelets (left column) and pseudo spectral velocity graphs (right column) of 1992 Landers Earthquake, Yermo Fire Station signal (a, b), 1999 Chi-Chi Taiwan Earthquake, TCU039 Station signal $(\mathbf{c}, \mathbf{d}), 1980$ Irpinia Earthquake $\left(M_{\mathrm{w}}=6.9\right)$, STN Station $\left(r_{\mathrm{ep}}=30.35 \mathrm{~km}\right)$ signal $(\mathbf{e}, \mathbf{f})$ and 1994 Northridge Earthquake $\left(M_{\mathrm{w}}=6.7\right)$, Rinaldi
Reveiving Station $\left(r_{\mathrm{ep}}=9.30 \mathrm{~km}\right)$ signal $(\mathbf{g}, \mathbf{h})$. Black, red, blue, and green signals are represent velocity waveform, Ricker wavelet, 4th-order Daubechies wavelet extracted by the algorithm of Shahi and Baker (2014) and extracted waveform by the algorithm of Chang et al. (2016), respectively. Vertical blue line represents the period of the pulse 
Table 1 Numerous signals with dissimilar impulsive results

\begin{tabular}{|c|c|c|c|c|c|c|c|}
\hline $\begin{array}{l}\text { Waveform } \\
\text { name }\end{array}$ & $\begin{array}{l}T_{\mathrm{p}} \\
\text { Shahi and Baker (2014) }\end{array}$ & PI & $\begin{array}{l}T_{\mathrm{p}} \\
\text { Chang et al. (2016) }\end{array}$ & $E_{\mathrm{p}}$ & $T_{\mathrm{p}}$ & $\begin{array}{l}\text { Waveform } \\
\text { energy }\end{array}$ & $\begin{array}{l}\text { Wavelet power } \\
\text { spectrum energy }\end{array}$ \\
\hline TCU078 & 0 & -0.71 & 1 & 0.50 & 3.60 & 74.15 & 63.79 \\
\hline Vineyard canyon $1 \mathrm{E}$ & 0 & -1.63 & 1 & 0.50 & 1.27 & 47.90 & 37.02 \\
\hline Brawley airport & 0 & -2.4 & 0 & 0.29 & 6.05 & 57.91 & 47.53 \\
\hline D08C & 3.89 & 1.90 & 0 & 0.30 & 0 & 29.74 & 23.41 \\
\hline $\mathrm{AQK}$ & 2.04 & 0.69 & 1.7 & 0.38 & 0 & 34.76 & 24.33 \\
\hline Pacoima dam & 0.78 & 7.69 & 0.7 & 0.38 & 0 & 39.78 & 19.19 \\
\hline KJMA & 1.09 & 5.82 & 1 & 0.35 & 0 & 38.34 & 19.24 \\
\hline Port Island & 2.7 & 5.94 & 2.1 & 0.39 & 0 & 32.35 & 18.21 \\
\hline
\end{tabular}

$T_{\mathrm{p}}$ is pulse period with studies that are used to calculate. $E_{\mathrm{p}}$ is explained in Eq. 3. Waveform energy and wavelet power spectrum energy are calculated as in Eqs. 4 and 7 depending on the position of the impulsive part, respectively. $T_{\mathrm{p}}$ gets 0 , if the signal is not found impulsive by the method that is used for the calculation. Waveforms are 25th of September 1999 Chi-Chi Taiwan Earthquake $\left(M_{\mathrm{w}}=6.3\right)$ TCU078 Station $\left(r_{\mathrm{ep}}=17.94 \mathrm{~km}\right)$, 28th of September 2004 Parkfield California Earthquake $\left(M_{\mathrm{w}}=6.0\right)$ Vineyard Canyon 1E Station $\left(r_{\mathrm{ep}}=18.76 \mathrm{~km}\right), 15$ th of October 1979 Imperial Valley California Earthquake $\left(M_{\mathrm{w}}=6.5\right)$ Brawley Airport Station $\left(r_{\mathrm{ep}}=43.15 \mathrm{~km}\right), 17$ th of May 1976 Gazli USSR Earthquake $\left(M_{\mathrm{w}}=6.8\right)$ Karakyr Station $\left(r_{\mathrm{ep}}=12.81 \mathrm{~km}\right), 21 \mathrm{st}$ of February 2011 Christchurch New Zealand Earthquake $\left(M_{\mathrm{w}}=6.2\right)$ D08C Station $\left(r_{\mathrm{ep}}=3.30 \mathrm{~km}\right)$, 6th of April 2009 L'Aquila Italy Earthquake $\left(M_{\mathrm{w}}=6.1\right)$ AQK Station $\left(r_{\mathrm{ep}}=1.76 \mathrm{~km}\right), 17$ th of January 1994 Northridge California Earthquake $\left(M_{w}=6.69\right)$ Pacoima Dam Station $\left(r_{\mathrm{ep}}=20.36 \mathrm{~km}\right)$, 16th of January 1995 Kobe Japan Earthquake $\left(M_{\mathrm{w}}=6.9\right) \mathrm{KJMA}\left(r_{\mathrm{ep}}=18.27 \mathrm{~km}\right)$ and Port Island $\left(r_{\mathrm{ep}}=\right.$ $19.25 \mathrm{~km}$ ) Stations, respectively

(2014) and Chang et al. (2016) (Fig. 5). Shahi and Baker (2014) identified 18 out of 26 of the signals as impulsive signal. Since Shahi and Baker (2014) are fitting full waveform on impulsive signals, it is not clear whether the impulsive part that was detected is the same region with our algorithm or not. Chang et al. (2016) identified 20 out of 26 of the signals as impulsive signal. Pulse periods that are calculated by our algorithm and Shahi and Baker (2014) and Chang et al. (2016) algorithms are close to each other.

Parts of signals that are considered by Shahi and Baker (2014) and Chang et al. (2016) and our method can be seen in Fig. 6. One can notice that these methods cover larger part of the waveform with respect to our method. This feature makes it harder to analyze the impulsive part of the waveform since it is spoiled by the non impulsive parts of the waveform.

We also focused on inconsistencies between previous methods and our method in terms of numerical results. One can notice that some signals are not identified as impulsive signal by one study whereas considered as impulsive in another one (Fig. 5a and b). Numerical results of Shahi and Baker (2014) (Eq. 2), Chang et al. (2016) (Eq. 3), and our method (Eq. 4) are explained in Table 1 .

Shahi and Baker (2014) were not able to identify some of the signals that are considered as impulsive by both Chang et al. (2016) and this study. PI is very close to the threshold of 0 on these examples. It is also valid for Chang et al. (2016). Threshold of 0.34 for Eq. 3 is almost exceeded at D08C Station. Brawley Airport Station is also just below the thresholds of Eq. 2 and Eq. 3, which gets a long pulse period by our study (Fig. 7). On the other hand, our method fails when the pulse period is short. In waveform energy parameter, which is the left side of the numerator of Eq. 4, the threshold is exceeded almost all non-impulsive signals, which are AQK, Pacoima Dam, KJMA, and Port Island Stations (Fig. 7). However, wavelet power spectrum energy (right side of the numerator of Eq. 4) is so small that it makes the signal not impulsive. Common feature of these signals that are identified as impulsive is the fact that they have 

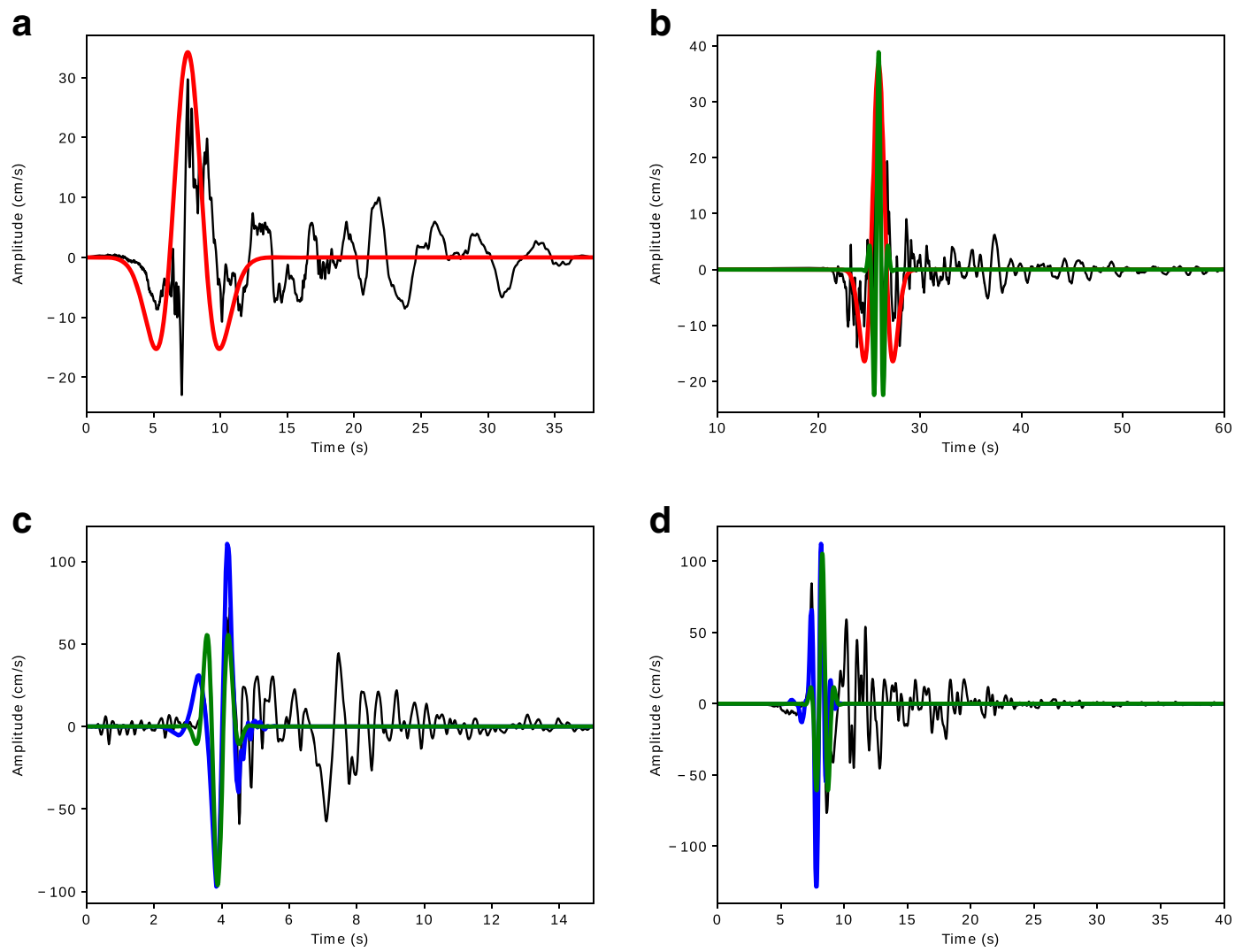

Fig. 7 Velocity waveforms and fitted wavelets of Brawley Airport (a), TCU078 (b), Pacoima Dam (c), and KJMA (d) stations. Colors represent the studies as in Fig. 6. Brawley Airport is labeled as impulsive by only our method $\left(T_{\mathrm{p}}=6.04\right)$, TCU078 has been found considered as impulsive by both our study

$\left(T_{\mathrm{p}}=3.60\right)$ and Chang et al. (2016) $\left(T_{\mathrm{p}}=1.00\right)$, Pacoima Dam and KJMA are identified as impulsive by both Shahi and Baker (2014) and Chang et al. (2016) with pulse periods of 0.78, 0.70 and $1.09,1.00$, respectively

very short impulses. One of the features of impulsive signals is their long periods (Section 1). Our method filters short period signals thanks to wavelet power spectrum energy.

\section{Conclusion}

In this study, we seek for an alternative way of identifying a pulse-shape signal. Combination of several methods that are created to look for the same features are used. The possibility of impulsive signals being located not on PGV but elsewhere is also taken into account. At the end, we have come up with the following conclusions:

1. Ricker wavelet analysis gives a higher resolution in the time domain, which is more suitable for determining the exact timing of the pulse.

2. A Ricker wavelet is better than Morlet wavelets for mimicking the pulse part of the earthquake signal based on residual analysis.

3. Our method is reproducing the spectral periods of the pulses, which makes the method convincing.

4. Most of the velocity pulses occurred at PGV. However, it is worth mentioning that pulses may occur also in other intervals of the signal.

5. This study has correlated with previous studies while expanding the information about the pulse shaped signal such as determining the pulse that occur outside the PGV region. 
Acknowledgments We would like to thank Assistant Professor Dr. Zhiwang Chang from School of Civil Engineering at Southwest Jiaotong University for sharing his pulse identification algorithm with us. We are grateful to anonymous referees for their vulnerable feedback on our work. We also would like to thank the National Research Institute for Earth Science and Disaster Resilience (NIED) for their website for K-NET and KiK-net (www.kyoshin.bosai.go.jp/, last accessed February, 2019) that makes it possible to access strong-motion seismographs in Japan, and GeoNet (www.geonet.org.nz/, last accessed February, 2019) for providing New Zealand strongmotion data. Python code of the method can be found at www. github.com/dertuncay/Pulse-Identification.

Funding information This study received financial support from the Italian Civil Protection (DPC) and Regional Civil Protection of Friuli-Venezia Giulia (FVG).

Open Access This article is distributed under the terms of the Creative Commons Attribution 4.0 International License (http:// creativecommons.org/licenses/by/4.0/), which permits unrestricted use, distribution, and reproduction in any medium, provided you give appropriate credit to the original author(s) and the source, provide a link to the Creative Commons license, and indicate if changes were made.

\section{Appendix 1: Ricker wavelet}

A Ricker wavelet is created by using Eq. 8,

$A=\left(1-2 \pi^{2} f^{2} t^{2}\right) e^{-\pi^{2} f^{2} t^{2}}$

$A, f$, and $t$ represent a Ricker wavelet, the frequency, and time stamp of the wavelet, respectively. An example of this wave can be seen in Fig. 8.

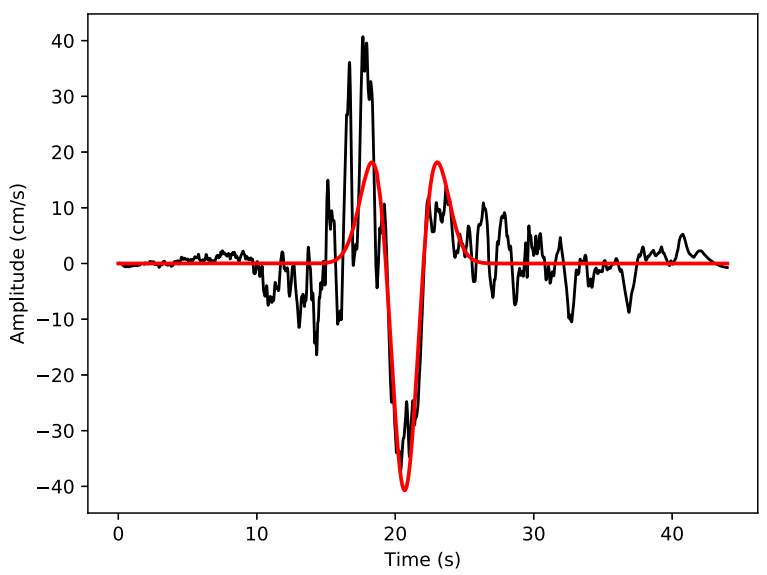

Fig. 81992 Landers earthquake, Yermo Fire Station velocity waveform (black) and Ricker wavelet (red) aligned at the position of PGV

\section{Appendix 2: Morlet wavelet}

A Morlet wavelet is constructed by multiplying a Gaussian wave with a sinusoidal wave. The sine wave is created as Eq. 9,

$S W=e^{\pi i f t}$

$S W, i, f$, and $t$ represent the sine wave, complex number, frequency and time stamps, respectively.

The Gaussian wave is created as Eq. 10,

Gaussian $=e^{\frac{-t^{2}}{2 s^{2}}}$

Gaussian, $t$, and $s$ indicate Gaussian wave, timestamp and width of the Gaussian, respectively. $S$ value sets the order of the wavelet.

Morlet $=S W \cdot$ Gaussian
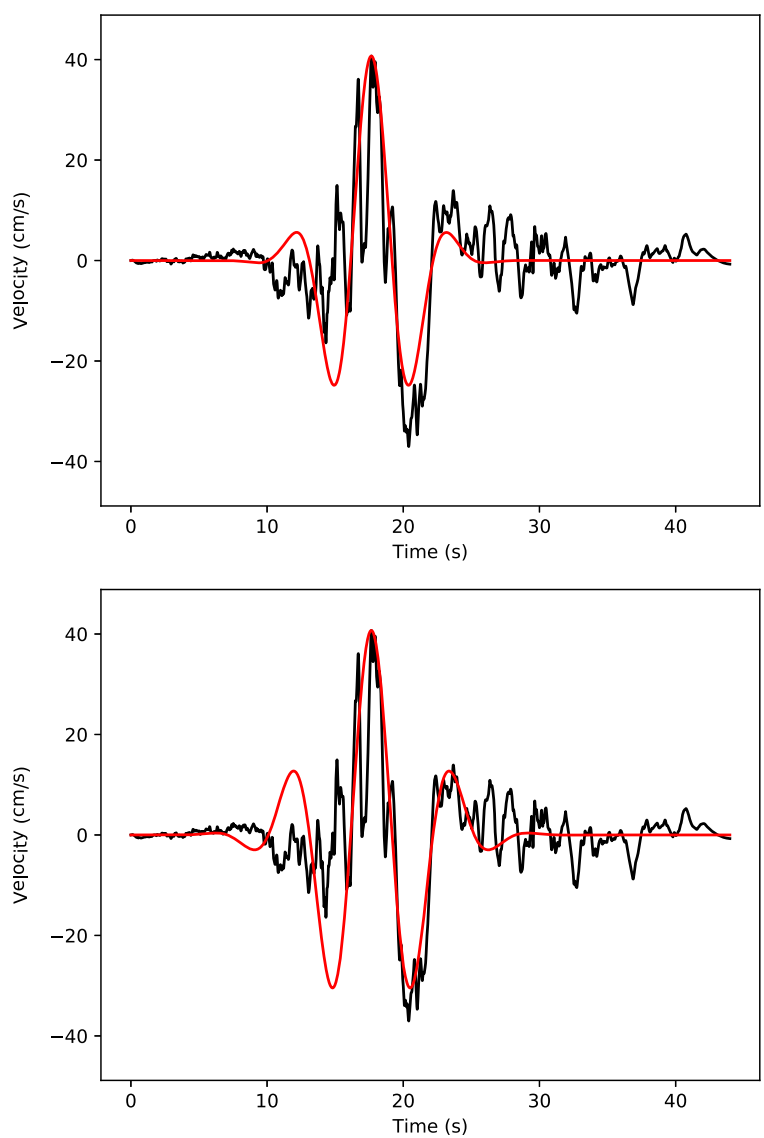

Fig. 9 3rd- and 4th-order Morlet wavelets (red) on 1992 Landers earthquake, Yermo Fire Station velocity waveform (black) and aligned at the position of PGV, respectively 
In Eq. 11, Morlet is the Morlet wavelet created by using the multiplication operation with $S W$ and Gaussian. The Morlet wavelet is used in 3rd and 4th orders. It can be seen in Fig. 9.

Abovementioned wavelets are subtracted from the original signal, separately. The wavelet that fits best, rendering the energy of the residual signal minimum, is recognized as the wavelet that mimicked the pulse most accurately.

\section{Appendix 3: Phase identification for Ricker wavelet}

Ricker wavelet that can represent the impulsive part of the waveform is rotated from 0 to $355^{\circ}$ on degrees.
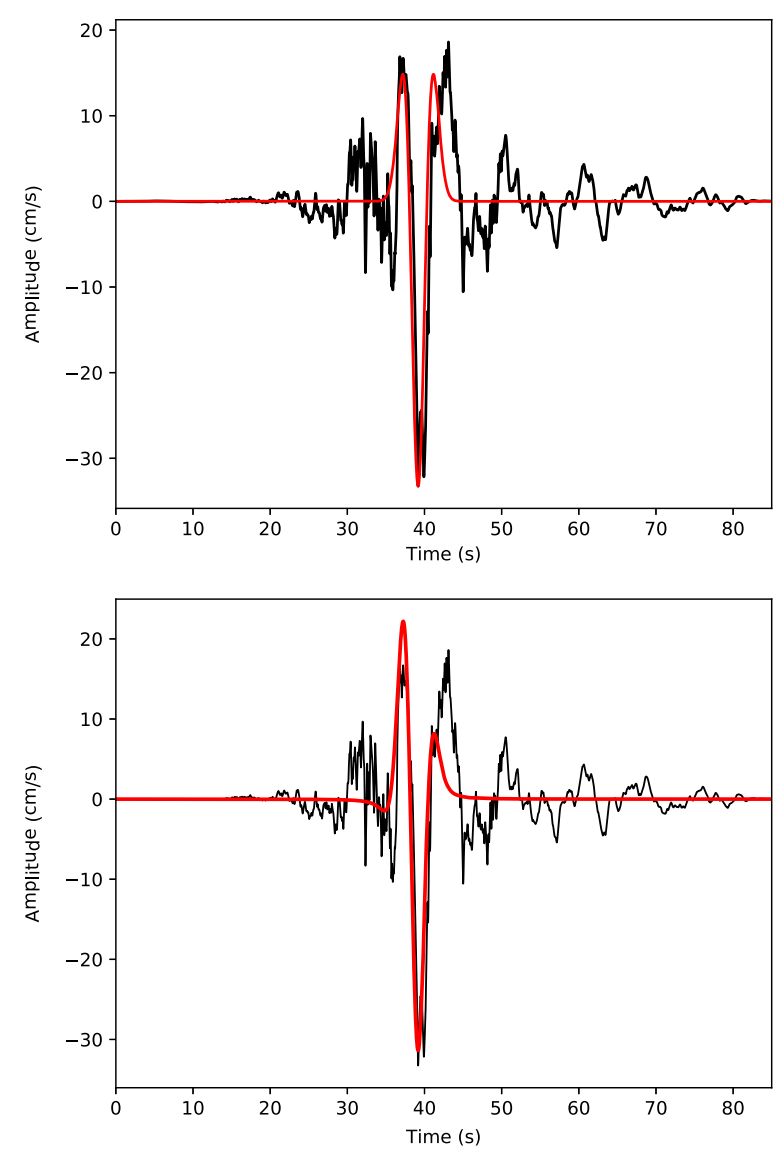

Fig. 101999 Chi-Chi Taiwan Earthquake, TCU046 Station signal $\left(r_{\mathrm{ep}}=78.17 \mathrm{~km}\right)$ and the Ricker wavelet without phase shifting (upper) and with phase shifting of $\theta=310^{\circ}$ (lower). Black and red waveforms are indicating the original velocity waveform and Ricker wavelet signals, respectively
In order to rotate the Ricker wavelet, first, the Hilbert transformation is applied on Ricker wavelet (Eq. 12).

$x+i y=F^{-1}(F(x) 2 U)$

In Eq. 12, $x$ and $i y$ represents the real and imaginary parts of the Hilbert transformed signal, respectively. $F(x)$ is the Fourier transform of the velocity waveform and $U$ is the unit step function. Then, the signal is rotated by using the real part, $x$, and imaginary part, $y$, of the transformed signal as in Eq. 13.

$R(\theta)=\cos (\theta) \times x-\sin (\theta) \times y$

Rotated Ricker wavelet is represented with $R(\theta)$ where $\theta$ is the rotation angle. Correlation coefficients are calculated between original waveform and rotated Ricker wavelet. Rotated wavelet with the highest correlation coefficient is considered as the representative of the impulsive part of the velocity waveform with a rotation angle. An example of a rotated Ricker wavelet can be seen in Fig. 10 .

\section{References}

Abrahamson N, Gregor N, Addo K (2016) Bc hydro ground motion prediction equations for subduction earthquakes. Earthq Spectra 32(1):23-44

Akkar S, Cheng Y (2016) Application of a monte-carlo simulation approach for the probabilistic assessment of seismic hazard for geographically distributed portfolio. Earthq Eng Struct Dyn 45(4):525-541

Akkar S, Yazgan U, Gülkan P (2005) Drift estimates in frame buildings subjected to near-fault ground motions. J Struct Eng 131(7):1014-1024

Alavi B, Krawinkler H (2001) Effects of near-fault ground motions on frame structures. John A. Blume Earthquake Engineering Center Stanford

Ancheta T, Bozorgnia Y, Darragh R, Silva W, Chiou B, Stewart J, Boore D, Graves R, Abrahamson N, Campbell K et al (2012) Peer nga-west2 database: a database of ground motions recorded in shallow crustal earthquakes in active tectonic regions. In: Proceedings, 15th World Conference on Earthquake Engineering

Anderson JC, Bertero VV (1987) Uncertainties in establishing design earthquakes. J Struct Eng 113(8):1709-1724

Bertero VV, Mahin SA, Herrera RA (1978) Aseismic design implications of near-fault san fernando earthquake records. Earthq Eng Struct Dyn 6(1):31-42

Boore DM, Stewart JP, Seyhan E, Atkinson GM (2014) Nga-west2 equations for predicting pga, pgv, and 5\% damped psa for shallow crustal earthquakes. Earthq Spectra 30(3):1057-1085

Bray JD, Rodriguez-Marek A (2004) Characterization of forward-directivity ground motions in the near-fault region. Soil Dyn Earthq Eng 24(11):815-828 
Chang Z, Sun X, Zhai C, Zhao JX, Xie L (2016) An improved energy-based approach for selecting pulse-like ground motions. Earthq Eng Struct Dyn 45(14):2405-2411

Ghaffarzadeh H (2016) A classification method for pulselike ground motions based on s-transform. Nat Hazards 84(1):335-350

Hall JF, Heaton TH, Halling MW, Wald DJ (1995) Near-source ground motion and its effects on flexible buildings. Earthq Spectra 11(4):569-605

Iwan W (1997) Drift spectrum: measure of demand for earthquake ground motions. J Struct Eng 123(4):397-404

Kalkan E, Kunnath SK (2006) Effects of fling step and forward directivity on seismic response of buildings. Earthq Spectra 22(2):367-390

Kardoutsou V, Taflampas I, Psycharis IN (2017) A new pulse indicator for the classification of ground motions. Bull Seismol Soc Am 107(3):1356-1364

Lanzano G, D'Amico M, Felicetta C, Puglia R, Luzi L, Pacor F, Bindi D (2016) Ground-motion prediction equations for region-specific probabilistic seismic-hazard analysis. Bull Seismol Soc Am 106(1):73-92

Luco N, Cornell CA (2007) Structure-specific scalar intensity measures for near-source and ordinary earthquake ground motions. Earthq Spectra 23(2):357-392

Luzi L, Puglia R, Russo E, D’Amico M, Felicetta C, Pacor F, Lanzano G, Çeken U, Clinton J, Costa G et al (2016) The engineering strong-motion database: a platform to access pan-european accelerometric data. Seismol Res Lett 87(4):987-997

Makris N, Black CJ (2004) Evaluation of peak ground velocity as a "good" intensity measure for near-source ground motions. J Eng Mech 130(9):1032-1044

Mavroeidis GP, Papageorgiou AS (2002) Near-source strong ground motion: characteristics and design issues. In: Proc. of the Seventh US National Conf on Earthquake Engineering (7NCEE), vol 21. Boston, Massachusetts, p 25

Mavroeidis GP, Papageorgiou AS (2003) A mathematical representation of near-fault ground motions. Bull Seismol Soc Am 93(3):1099-1131
Mavroeidis G, Dong G, Papageorgiou A (2004) Near-fault ground motions, and the response of elastic and inelastic single-degree-of-freedom (sdof) systems. Earthq Eng Struct Dyn 33(9):1023-1049

Mena B, Mai PM (2011) Selection and quantification of nearfault velocity pulses owing to source directivity. Georisk $5(1): 25-43$

Menun C, Fu Q (2002) An analytical model for near-fault ground motions and the response of sdof systems. In: Proceedings, 7th US National Conference on Earthquake Engineering, p 10

Pacor F, Paolucci R, Luzi L, Sabetta F, Spinelli A, Gorini A, Nicoletti M, Marcucci S, Filippi L, Dolce M (2011) Overview of the Italian strong motion database itaca 1.0. Bull Earthq Eng 9(6):1723-1739

Shahi SK, Baker JW (2014) An efficient algorithm to identify strong-velocity pulses in multicomponent ground motions. Bull Seismol Soc Am 104(5):2456-2466

Somerville PG (2003) Magnitude scaling of the near fault rupture directivity pulse. Phys Earth Planet Inter 137(14):201-212

Somerville PG (2005) Engineering Characterization of near fault ground motions. In: Proc NZSEE 2005 Conf

Somerville PG, Smith NF, Graves RW, Abrahamson NA (1997) Modification of empirical strong ground motion attenuation relations to include the amplitude and duration effects of rupture directivity. Seismol Res Lett 68(1):199-222

Spudich P, Chiou BS (2008) Directivity in nga earthquake ground motions: Analysis using isochrone theory. Earthquake Spectra 24(1):279-298

Torrence C, Compo GP (1998) A practical guide to wavelet analysis. Bull Am Meteorol Soc 79(1):61-78

Yang D, Wang W (2012) Nonlocal period parameters of frequency content

Publisher's note Springer Nature remains neutral with regard to jurisdictional claims in published maps and institutional affiliations. 